\title{
Globular glial tauopathy, a newly recognized white matter tauopathy, with depression/anxiety disorder: report and review of classification
}

\author{
Maher Kurdi ${ }^{1}$, Badrah Saeed Alghamdi ${ }^{2,3}$, Jeremy Parfitt ${ }^{4}$, Lee Cyn Ang ${ }^{4}$ \\ ${ }^{1}$ Department of Pathology, Faculty of Medicine, Rabigh, King Abdulaziz University, Saudi Arabia, ${ }^{2}$ Department of Physiology, \\ Neuroscience Unit, Faculty of Medicine, King Abdulaziz University, Jeddah, Saudi Arabia, ${ }^{3}$ Pre-Clinical Research Unit, King Fahad \\ Medical Research Center, King Abdulaziz University, Jeddah, Saudi Arabia, ${ }^{4}$ Department of Pathology, Schulich School of Medicine \\ and Dentistry, Western University, London, Canada
}

\begin{abstract}
Several studies have recently described 4-repeat tauopathies that are characterized by the presence of globular glial inclusions. These inclusions are astrocytic or oligodendroglial, show extensive white matter involvement, and have a wide range of neuropathological and clinical presentations. Globular glial tauopathy (GGT) is classified into three subtypes according to clinical manifestations. Type I is characterized by frontotemporal lobar degeneration and represents a group of diseases with diverse clinical and histopathological features. Some of these disorders are associated with abnormal microtubule-associated protein tau gene. Type II presents as motor impairment due to pyramidal involvement, whereas type III is a combination of the features of types I and II. This report describes a rare case of GGT that manifested as depression and anxiety and demonstrates its neuropathological features. We have also compared the features of this case with those of previously reported cases and have revisited the classification.
\end{abstract}

Key words: frontotemporal lobe degeneration, tauopathies, 4-repeat tauopathies, white matter tauopathy, globular glial tauopathy.

\section{Introduction}

Frontotemporal lobar degeneration (FTLD) represents a group of diseases characterized by the degeneration of the frontal and temporal lobes and diverse clinical and histopathological features. FTLD with tauopathies shows abnormal accumulation of hyperphosphorylated tau protein, which is encoded by microtubule-associated protein tau (MAPT) on chromosome $17[1,2,4,10]$. Based on biochemical and molecular characteristics, tauopathies are classified into 3-repeat (3R) and 4-repeat (4R) isoforms [6]. The $4 R$ isoform is seen in progressive supranuclear palsy (PSP), corticobasal degeneration (CBD), argyrophilic grain disease (AGD), and FTLD-MAPT [6]. Globular glial tauopathy (GGT) is a newly described spectrum of disorders that shares similarities with the $4 R$ subtype, but with predominant white matter involvement [6]. Several recent studies have provided insights on $4 R$ tauopathies that show widespread 
presence of globular glial inclusions (GGIs) on histology [6]. Individuals with GGT present with a wide range of clinicopathological features and are classified into three groups based on clinical presentation (Fig. 1) [1]. Type I shows FTLD, i.e., sporadic multiple system tauopathy with presenile dementia. Type II is characterized by a greater involvement of the motor cortex with corticospinal tract lesions [i.e., motor neuron disease (MND)]. Type III shows a combination of features of both types I and II, i.e., FTLD-MND [1]. The severity of clinical manifestations is directly correlated with the distribution of GGIs. However, variable clinical manifestations and neuropathological features were reported in GGT patients with a recently described GGT case presented clinically as Alzheimer disease $[8,17]$. Here, we describe an unusual case of a patient who presented with depression and anxiety with histologically confirmed GGIs.

\section{Clinical summary}

A 65-year-old male was found dead in his home. His medical history included chronic alcohol dependence and mixed depression/anxiety disorder since the age of 30 years. He had multiple hospitalizations for alcohol overdose and withdrawal seizures. However, there was no history of hallucination, delusion, or associated motor symptoms. He had previously denied any history of dementia or behavioural symptoms. No family history of psychiatric disorders was documented. Socially, he was living alone and had been divorced twice.

A mini-mental status examination, conducted 2.5 years before his death during hospitalization for alcohol withdrawal, had a recorded result of 27/30 [Clinical Institute Withdrawal Assessment for Alcohol (CIWA) scale]. He appeared cognitively intact. Additionally, his language was coherent, and his thoughts were organized. Because documentation revealed two episodes of attempted suicide using hydromorphone overdose and self-injury after heavy drinking, he had been referred to a concurrent disorder program for behavioural modification; however, he never attended the program sessions.

A general autopsy was performed upon request by the coroner. There was no evidence of traumatic injury, and the toxicology result was negative. The cause of his sudden death was related to his chronic alcoholism which is associated with withdrawal

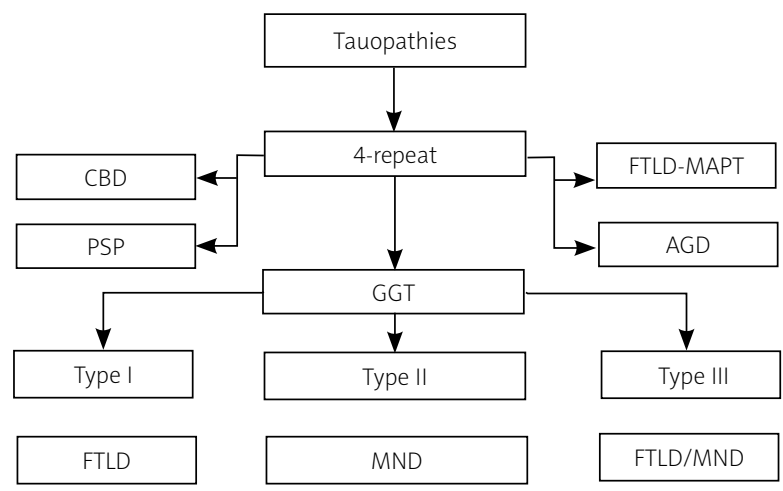

Fig. 1. Classification of $4 R$ tauopathies and globular glial tauopathy (GGT) subtypes. CBD - corticobasal degeneration, FTLD - frontotemporal dementia, MAPT - microtubule-associated protein tau, PSP - progressive supranuclear palsy, AGD - argyrophilic grain disease, MND - motor neuron disease.

seizures, cardiac arrhythmias, and metabolic disturbances.

\section{Pathological findings}

Examining the unfixed brain, weighed $1367 \mathrm{~g}$, at autopsy showed no evidence of traumatic brain injury. Gross examination of the entire brain and brainstem with the cerebellum was unremarkable. The brain-cut protocol for a coroner's case was executed but no significant abnormalities were noted on coronal sections, particularly in the mesial temporal structures (Fig. 2). Representative slices were retained and fixed in $10 \%$ formalin for 10 days for neuropathological examination and the rest of the brain was returned with the body.

Histopathological examination of cortical and subcortical structures was performed using light microscopy. Luxol fast blue haematoxylin-eosin (LFB/HE) histochemical stain and tau-paired-helical-filament (PHF) immunohistochemistry were predominantly used for neuropathological assessment. Tau-PHF was prepared by tau-mouse monoclonal antibodies from Fisher ENMN1020, antigen retrieval at high $\mathrm{pH}, 97^{\circ} \mathrm{C}$ for $20 \mathrm{~min}, 1$ : 4000 dilution, EnVision Flex polymer detection system without amplification and DAB visualization for $10 \mathrm{~min}$. In addition, modified Bielschowsky histochemical stain and immunostain for $\beta$-amyloid, $\alpha$-synuclein, and TDP43 were performed. 


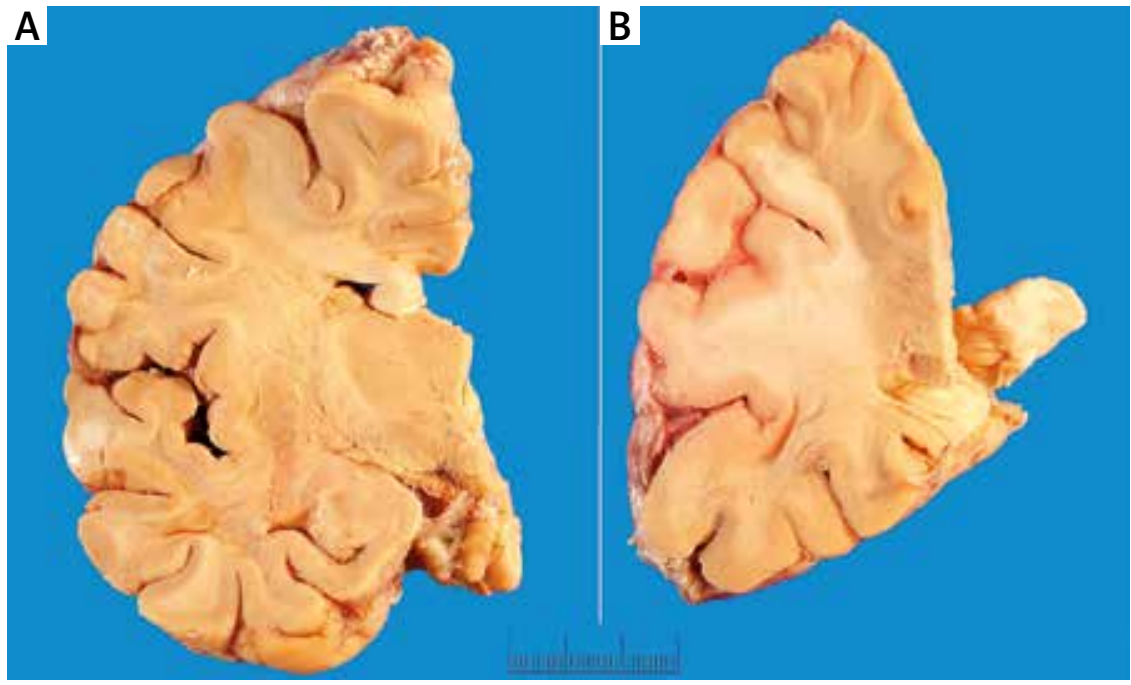

Fig. 2. A, B) Gross examination of coronal sections of the frontotemporal region was unremarkable.

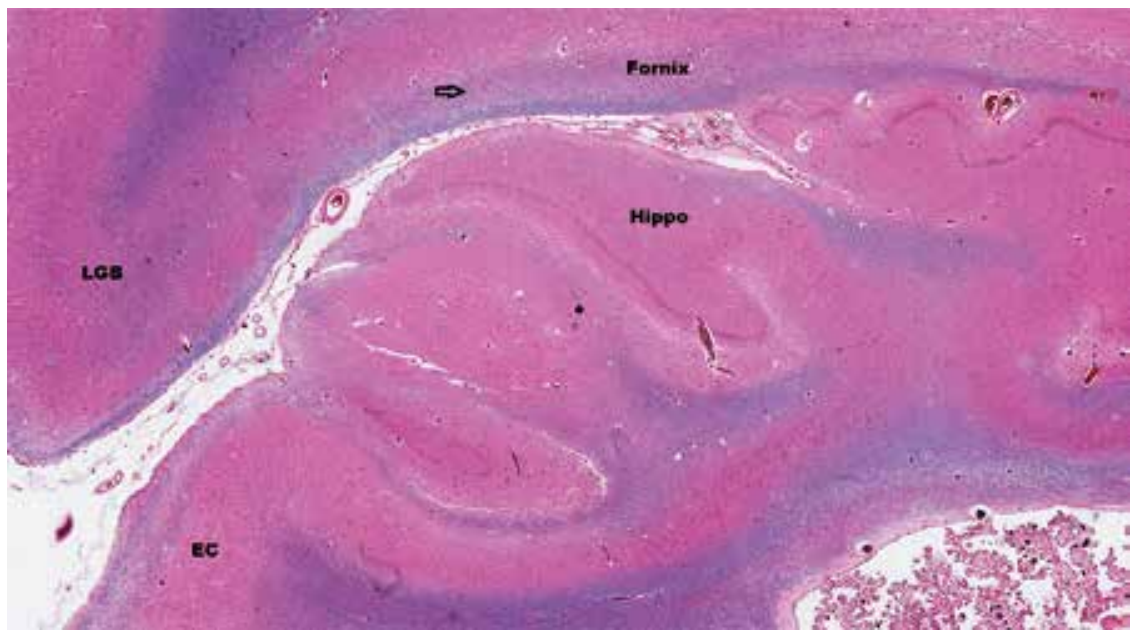

Fig. 3. A section showing the hippocampus and fornix, with the fornix showing minimal loss of white matter. Fornix, Hippo - hippocampus, LGB - lateral geniculate body, EC - entorhinal cortex. Scale bar $=600 \mu \mathrm{m}$.

There was a minimal loss of myelin in the temporal white matter and fornix secondary to degenerative changes (Fig. 3). Tau-PHF staining showed rare pentangles in the pre-transentorhinal cortex. There was a significant amount of globular glial cytoplasmic inclusions, both globular oligodendroglial inclusions (GOIs) and globular astrocytic inclusions (GAls), involving the frontal and temporal white matter (Figs. 4 and 5), hippocampi, amygdala and fornix. These inclusions were also seen in internal and external capsules, and anterior commissure. The general anatomical distribution of these inclusions was consistent with that previously described for dementia with GGIs. No amyloid plaques, includ- ing neuritic plaques were identified in the cortical sections. No other types of inclusions were seen with alpha-synuclein or TDP-43 immunostaining.

\section{Discussion}

Frontotemporal lobar degeneration comprises a group of neurological diseases characterized by atrophy and degeneration of both frontal and temporal cortices. Patients may present with dementia and progressive deterioration in behaviour, personality, cognition, and language dysfunction [5]. A few patients may also present with semantic dementia or non-fluent aphasia. 


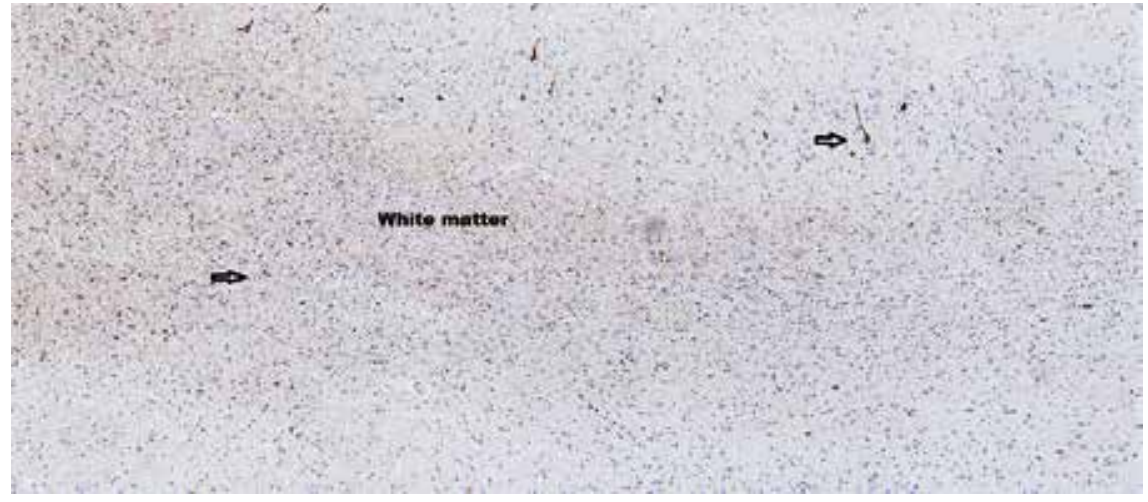

Fig. 4. A section showing extensive tangle inclusions in the cortical and subcortical white matter of the inferior temporal region indicated by arrows (Tau-PHF staining). Scale bar $=300 \mu \mathrm{m}$.

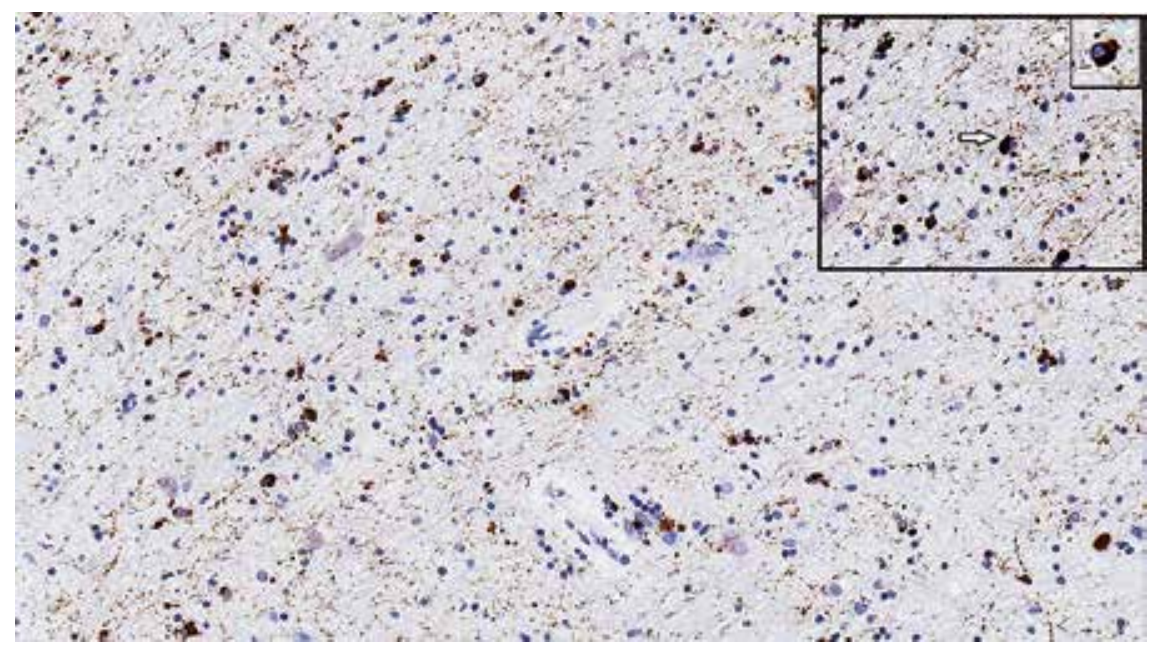

Fig. 5. A section from the temporal white matter shows significant globular glial cytoplasmic inclusions indicated by an arrow (globular oligodendroglial inclusions and globular astrocytic inclusions). Scale bar = $200 \mu \mathrm{m}$.

Tauopathies are defined by the presence of ectopic aggregates of hyperphosphorylated tau in neurons and glial cells, oligodendrocytes and include FTLD associated with abnormal MAPT. MAPT that encodes tau which promotes the assembly and stability of microtubules, has 14 coding exons, and it undergoes alternative splicing at exons 2, 3, and 10 to produce six protein isoforms with either 3 or 4 repeat regions in their microtubule-binding domains; these variants are accordingly named $3 R$ or $4 R$ tau isoforms [7]. Both isoforms are expressed at nearly the same level in a normal adult human brain, but their levels are altered in tauopathies $[12,18]$.

The 4R tauopathy has been demonstrated in PSP, CBD, FTLD-MAPT, and AGD (Fig. 5) [17]. Recent studies have reported another manifestation of this isoform, which is characterized by GGI $[1-3,9,14-16,18]$. The severity of clinical symptoms in these patients is associated with the distribution of underlying tau pathology [1]. Some of these cases present with frontotemporal dementia, and the rest of the cases exhibit varying features of pyramidal and extrapyramidal disorders. In 1998, Molina et al. [15] described two types of glial cytoplasmic inclusions in a patient with moderate frontotemporal atrophy and primary progressive aphasia.

Globular glial tauopathy, also known as white matter tauopathy, is a very rare $4 \mathrm{R}$ tauopathy histologically characterized by tau-positive GGls and extensive white matter involvement. The diameters of these inclusions (including GOls and/or GAls) range between 8 and $20 \mu \mathrm{m}$ [14]. They can be identified using H\&E staining or silver impregnation tech- 
niques (Bielschowsky or Gallyas method) but are more evident with tau immunohistochemistry.

In 2008, Kovacs et al. $[13,14]$ reported the first comprehensive assessment of seven patients with GGIs, and they named this condition white matter tauopathy with globular glial inclusions. All cases showed inclusions, predominantly in oligodendrocytes. Ahmed et al. [1] performed a comparative clinicopathological and biochemical study of two patients with neurodegeneration and identified both GOls (positive for Gallyas stain) and GAls (negative for Gallyas stain). Oligodendrocytes are heavily involved (i.e. GOls) and predominate in the development of white matter lesions. Furthermore, the presence of myelin pallor, axonal loss, and gliosis suggest a scenario wherein GGT can be regarded as primary oligodendrogliopathy [2]. In our case, LFB/HE staining showed a rarefied white matter change in the temporal lobe and fornix, along with marked chronic gliosis. We used Tau-PHF to highlight the glial inclusions. GGT can be grouped with PSP/ $\mathrm{CBD}$, and these pathologies share astrocytic inclusion morphology and tau structure [1]. Nonetheless, it can be differentiated from other $4 R$ tauopathies despite the histological similarities because balloon cells are always present in CBD and AGD but are absent in PSP and GGT [7]. Tau-positive neuritic changes are evident in the frontal and temporal white matter in CBD and the absence of thread-like processes in the white matter makes its diagnosis unlikely [7]. Tau-positive coiled bodies are present in all 4-R tauopathies, namely, PSP, CBD, and AGD [7]. Generally, the Gallyas silver stain can be used as a diagnostic tool to help distinguish between GGT and PSP; however, it is not useful in certain cases in which the evaluation of astrocytic pathology is essential for differentiation of the two entities [11]. However, GGT can be distinguished from FTLD-MAPT because the latter shows Gallyas-positive astrocytic inclusions [1]. The lack of consistent terminology for cases with GGls and absence of standard molecular neuropathology has impeded their accurate classification; however, an international consensus committee has proposed a sub-stratification strategy with three clinicopathological subtypes of GGT [1]. This classification is based on regional distribution, frequency of the inclusions and associated clinical features (Fig. 5).

Our patient presented with mixed depression/ anxiety symptoms and chronic alcoholism, and to the best of our knowledge, depression and anxiety have not been adequately emphasized as early presentation in previous reports. Although it would be erroneous for us to conclude that our patient's depression/anxiety disorder was solely owing to GGT, the fact that the patient did not recover from the death of his son even after a prolonged period certainly suggests the presence of other underlying factors, one of which could be GGT. This case is interesting owing to the absence of clinical dementia and the major presentation of depression and anxiety, despite the histopathological findings confirming GGT subtype I according to the classification detailed by the international consensus committee. Kovac et al. [14] described a case of GGT in which the patient presented with depression and anxiety. Similarly, Tacik et al. [18] reported that depression and anxiety were the initial presenting symptoms in a patient who was subsequently diagnosed with behavioral variant frontotemporal dementia (bvFTD). The neuropathological features and anatomic distribution were consistent with those previously described for GGT with white matter involvement.

Kovacs et al. [14] found no mutations in the exons and adjoining intronic regions of MAPT in their cases, whereas the $\mathrm{H} 1 / \mathrm{H} 1$ genotype was found in 4 cases. Ahmed et al. [1] also found no mutations in 25 of the 30 GGT cases reported in the literature that have been screened for mutations in MAPT [1]. Tacik et al. [18] found a novel MAPT mutation (c.951G>C, p.K317N) in one of the studied cases. Recombinant 3R- and 4R-tau bearing the p.K317N mutation show decreased $3 R$ tau but increased $4 R$ tau filament assembly.

\section{Conclusions}

Globular glial tauopathy is a newly described entity that falls within the group of $4 R$ tauopathies with predominant white matter involvement. It has been classified into three subtypes. Based on the symptoms in our case and those of previously reported cases, this variant of GGT with depression/anxiety disorders deserves further distinction. Owing to the overlapping microscopic features among the various GGT subtypes, we recommend that future criteria should focus on specific clinical presentations, in addition to the detailed regional distribution of inclusions and neurodegeneration with the support of genetic analysis. 


\section{Ethics approval}

This case report was completed in alignment with the London Health Science Center guidelines for case reports.

\section{Disclosure}

\section{The authors report no conflict of interest.}

\section{References}

1. Ahmed Z, Bigio EH, Budka H, Dickson DW, Ferrer I, Ghetti B, Giaccone G, Hatanpaa KJ, Holton JL, Josephs KA, Powers J, Spina S, Takahashi H, White CL 3rd, Revesz T, Kovacs GG. Globular glial tauopathies (GGT): consensus recommendations. Acta Neuropathol 2013; 126: 537-544.

2. Ahmed Z, Doherty KM, Silveira-Moriyama L, Bandopadhyay R, Lashley T, Mamais A, Hondhamuni G, Wray S, Newcombe J, O'Sullivan SS, Wroe S, de Silva R, Holton JL, Lees AJ, Revesz T. Globular glial tauopathies (GGT) presenting with motor neuron disease or frontotemporal dementia: an emerging group of 4-repeat tauopathies. Acta Neuropathol 2011; 122: 415-428.

3. Berry RW, Quinn B, Johnson N, Cochran EJ, Ghoshal N, Binder LI. Pathological glial tau accumulations in neurodegenerative disease: review and case report. Neurochem Int 2001; 39: 469 479.

4. Bigio EH, Lipton AM, Yen SH, Hutton ML, Baker M, Nacharaju P, White CL 3rd, Davies P, Lin W, Dickson DW. Frontal lobe dementia with novel tauopathy:sporadic multiple system tauopathy with dementia. J Neuropathol Exp Neurol 2001; 60: 328-341.

5. Boxer AL, Miller BL. Clinical features of frontotemporal dementia. Alzheimer Dis Assoc Disord 2005; 19: S3-6.

6. Chung DC, Carlomagno Y, Cook CN, Jansen-West K, Daughrity L, Lewis-Tuffin LJ, Castanedes-Casey M, DeTure M, Dickson DW, Petrucelli L. Tau exhibits unique seeding properties in globular glial tauopathy. Acta Neuropathol Commun 2019; 7: 36-47.

7. Dickson DW, Ahmed Z, Algom AA, Tsuboi Y, Josephs KA. Neuropathology of variants of progressive supranuclear palsy. Curr Opin Neurol 2010; 23: 394-400.

8. Erro ME, Zelaya MV, Mendioroz M, Larumbe R, Ortega-Cubero S, Lanciego JL, Lladó A, Cabada T, Tuñón T, García-Bragado F, Luquin MR, Pastor P, Ferrer I. Globular glial tauopathy caused by MAPT P301T mutation: clinical and neuropathological findings. J Neurol 2019; 266: 2396-2405.

9. Ferrer I, Hernández I, Boada M, Llorente A, Rey MJ, Cardozo A, Ezquerra M, Puig B. Primary progressive aphasia as the initial manifestation of corticobasal degeneration and unusual tauopathies. Acta Neuropathol 2003; 106: 419-435.

10. Fu YJ, Nishihira Y, Kuroda S, Toyoshima Y, Ishihara T, Shinozaki M, Miyashita A, Piao YS, Tan CF, Tani T, Koike R, Iwanaga K, Tsujihata M, Onodera O, Kuwano R, Nishizawa M, Kakita A, Ikeuchi T, Takahashi H. Sporadic four-repeat tauopathy with frontotemporal lobar degeneration, Parkinsonism, and motor neuron disease: a distinct clinicopathological and biochemical disease entity. Acta Neuropathol 2010; 120: 21-32.
11. Gallyas F. Silver staining of Alzheimer's neurofibrillary change by means of physical development. Acta morphol Acad Sci Hung 1971; 19: 1-8.

12. Goedert M, Spillantini MG, Potier MC, Ulrich J, Crowther RA. Cloning and sequencing of the CDNA encoding an isoform of microtubule-associated protein tau containing four tandem repeats: differential expression of Tau protein mRNAs in human brain. EMPO J 1989; 8: 393-399.

13. Josephs KA, Katsuse O, Beccano-Kelly DA, Lin WL, Uitti RJ, Fujino Y, Boeve BF, Hutton ML, Baker MC, Dickson DW. Atypical progressive supranuclear palsy with corticospinal tract degeneration. J Neuropathol Exp Neurol 2006; 65: 396-405.

14. Kovacs GG, Majtenyi K, Spina S, Murrell JR, Gelpi E, Hoftberger R, Fraser G, Crowther RA, Goedert M, Budka H, Ghetti B. White matter tauopathy with globular glial inclusions: a distinct sporadic frontotemporal lobar degeneration. J Neuropathol Exp Neurol 2008; 67: 963-975.

15. Molina JA, Probst A, Villanueva C, Jiménez-Jiménez FJ, Madero S, Torres N, Bermejo F. Primary progressive aphasia with glial cytoplasmic inclusions. Eur Neurol 1998; 40: 71-77.

16. Powers JM, Byrne NP, Ito M, Takao M, Yankopoulou D, Spillantini MG, Ghetti B. A novel leukoencephalopathy associated with tau deposits primarily in white matter glia. Acta Neuropathol 2003; 106: 181-187.

17. SantaCruz KS, Rottunda SJ, Meints JP, Bearer EL, Bigio EH, McCarten JR. A case of globular glial tauopathy presenting clinically as alzheimer disease. Alzheimer Dis Assoc Disord 2015; 29: 82-84.

18. Tacik P, DeTure M, Lin WL, Sanchez Contreras M, Wojtas A, Hinkle KM, Fujioka S, Baker MC, Walton RL, Carlomagno Y, Brown PH, Strongosky AJ, Kouri N, Murray ME, Petrucelli L, Josephs KA, Rademakers R, Ross OA, Wszolek ZK, Dickson DW. A novel tau mutation, p.K317N, causes globular glial tauopathy. Acta Neuropathol 2015; 130: 199-214. 